\title{
Nachvollzug Zusammenführung SGAM und SGIM
}

\section{Michael Barnikola, Hanspeter Kuhn ${ }^{b}$}

${ }^{a}$ Dr. iur., Rechtsdienst FMH; ${ }^{b}$ Fürsprecher, Abteilungsleiter, Abteilung Rechtsdienst FMH

1 Nur in den Rubriken «Notfalldienst ärztlich» und «Telemedizin» können sich wie bisher ebenfalls juristische Personen eintragen lassen.

2 Wie bisher sind die kant. Ärztegesellschaften zuständig für die Regelung von Inserategrösse, Fettdruck, Zulassung von Farbenmix etc. Für inhaltliche Angaben siehe die Richtlinien Information und Werbung (Anhang 2 zur Standesordnung der FMH) und die Empfehlun gen FMH/SIWF von November 2015 zur Ausschreibung von akademischen Bezeichnungen, Facharzttiteln und anderen ärztlichen Qualifikationen, Dienstleistungsangeboten, nichtärztlichen Qualifikationen, Nachdiplomstudien und Mitgliedschaften (www.fmh ch $\rightarrow$ Service $\rightarrow$ Recht).

Korrespondenz: Sekretariat Rechtsdienst FMH

Tel. 0313591111

Fax 0313591112

lex[at]fmh.ch

Patienten suchen im Telefonbuch und immer häufiger auf Internetportalen nach einer Ärztin oder einem Arzt. Um die Suche zu vereinfachen und transparenter zu machen, hat die FMH mit der Swisscom Directories AG die Publikationsbestimmungen präzisiert. Ab 2017 gibt es wesentliche Neuerungen zu Gunsten von Hausärztinnen und Hausärzten.

Die Vereinbarung zwischen der FMH und der Swisscom Directories AG wurde revidiert. Ab 2017 wird es in den gedruckten Telefonbüchern und auf den Internetportalen search.ch und local.ch bei den Ärzteeinträgen folgende Neuerungen geben:

\section{Rubriken Allgemeinmedizin und Innere Medizin zusammengeführt}

Neu werden die Rubriken "Allgemeinmedizin" und «Innere Medizin» unter der Rubrik «Allgemeine Innere Medizin» zusammengefasst. Der Rubrikname entspricht damit der aktuellen Titelnomenklatur der Medizinalberufeverordnung. Die Rubriken "Allgemeinmedizin» und «Innere Medizin» werden aufgehoben. An Stelle der bisherigen Rubriktitel wird der Hinweis siehe unter «Allgemeine Innere Medizin» aufgeführt.

Die Zusammenführung der Rubriken erfolgt automatisch durch Swisscom Directories AG in der Zeit ab 1. bis 28. Februar 2017. Sie als Ärztin oder Arzt müssen nichts unternehmen. Viele Grundversorger haben sich bisher in beiden Rubriken eingetragen, um sicher gefunden zu werden. Aufgrund der Zusammenlegung der beiden Rubriken können sie Gebühren sparen.

\section{Ergänzung «Hausärztin/Hausarzt»}

Wer als Hausärztin oder Hausarzt tätig ist, kann seinen Eintrag mit «Hausärztin» oder «Hausarzt» ergänzen lassen. Falls Sie diese Ergänzung wünschen, können Sie dies Swisscom Directories AG online (unter https://info.local.ch $\rightarrow$ Ihr Eintrag $\rightarrow$ Eintrag ändern) oder telefonisch über die kostenlose Kundendienstnummer 0800868086 mitteilen.

Für Patienten ist damit eine einfache Online-Suche nach einem Hausarzt oder einer Hausärztin möglich.

\section{Keine Werbung von Instituten unter der Rubrik «Ärzte»}

Gemäss der Standesordnung und der Vereinbarung mit der Swisscom Directories AG sind Einträge von juristischen Personen unter dem Stichwort «Ärzte» nicht erlaubt (Ziff. 3.4.1 in Anhang 2 der Standesordnung). Um einer Umgehung dieser Regelung durch Inserate vorzubeugen, wurde die Vereinbarung zwischen der FMH und der Swisscom Directories AG wie folgt ergänzt (kursiv): Unter dem Stichwort Ärzte ${ }^{1}$ «... können sich ausschliesslich natürliche Personen (also keine Institutionen) eintragen lassen. Dies wird auch in Bezug auf Werbung in den Telefonbüchern berücksichtigt (keine Werbung von Institutionen unter der Rubrik Ärzte).»

\section{Beispielinserat $^{2}$}

Allgemeine Innere Medizin, Ärzte

Dr. med. Johanna Musterfrau

Fachärztin für Allgemeine Innere Medizin $(\mathrm{CH})$,

Mitglied FMH, Hausärztin

Öffnungszeiten:

Mo-Mi 8-12 + 13.30-17.30 Uhr, Do 8-12 +16-20 Uhr, Fr 8-15 Uhr Musterstrasse 1

1000 Musterstadt

Telefon $0111111111^{*}$

Telefax 01111111 12*

[* Wünscht keine Werbung] 